\title{
Der Bundesvorstand berichtet
}

\section{Sitzungen am 19. Februar 2011 und am 25. Juni 2011 in Berlin}

\section{Jutta Wagner}

Ein letztes Mal vor dem Ende meiner sechsjährigen Amtszeit als Präsidentin des djb berichte ich hier von unseren Vorstandssitzungen.

In beiden Sitzungen wurde über Nachfolgefragen gesprochen - meine und die in weiteren Ämtern. Am 24. September 2011 in Potsdam werden das Präsidium, die Beisitzerinnen und die Delegierten neu gewählt. Einige Kandidatinnen haben die Gelegenheit ergriffen, sich in der djbZ 3/2011 (S. 137) vorzustellen, darunter meine bisherige Vizepräsidentin Ramona Pisal, die für das Amt der Präsidentin kandidieren wird.

Am 19. Februar 2011, in der ersten Sitzung des Jahres, wurde über Finanzen und verschiedene organisatorische Themen diskutiert und beschlossen, darunter Beschlüsse über den Wirtschaftsplan des Jahres, die Höhe der Zuweisungen an die Landesverbände und Regionalgruppen und - leider - die Ausschlüsse aus dem djb wegen Nichtzahlung von Mitgliedsbeiträgen in drei Jahren.

Immer noch sind trotz Zahlungserinnerung für 2011 die Außenstände vergleichsweise hoch. Ich bitte daher herzlich und dringend alle Mitglieder, deren Jahresbeitrag noch aussteht, um Zahlung. Unsere Arbeit hängt davon ab.

Vieles zum Thema verbandsinterne Finanzen ist, seit der djb sich im Mai 2010 der „Initiative Transparente Zivilgesellschaft" von Transparency International Deutschland e.V. angeschlossen hat, standardmäßig auf der Webseite (http://www. djb.de/extras/Transparenz/) abrufbar. Die Initiative hat sich auf wesentliche Parameter zur Schaffung von Transparenz geeinigt und wir erfüllen unsere Selbstverpflichtung, zehn präzise benannte, relevante Informationen zur Organisation leicht auffindbar und in einem bestimmten Format der breiten Öffentlichkeit zugänglich zu machen. Dazu gehören auch Angaben zur Mittelverwendung. Die Zuweisungen an die Landesverbände werden schon seit 2007 auf der Homepage dokumentiert (http://www.djb.de/verein/Finanzstruktur/ Selbstverwaltung/). Der aktuellen Berechnung liegt ein Bundesvorstandsbeschluss vom 16. Januar 2010 für 2010 und 2011 zugrunde.

Auch die Mitgliedschaften des djb in anderen Vereinigungen sind ein regelmäßiger Punkt auf unseren Tagesordnungen. Nach intensiver Diskussion erfolgte am 19. Februar 2011 der einstimmige Beschluss, der Deutschen Stiftung für internationale rechtliche Zusammenarbeit (IRZ-Stiftung, http://www.irzstiftung.de/) beizutreten. Die IRZ-Stiftung fördert und pflegt die internationale Zusammenarbeit auf dem Gebiet des Rechts im Sinn der Völkerverständigung. Insbesondere unterstützt sie ausländische Staaten beim Übergang von der Planwirtschaft in die soziale Marktwirtschaft im Bereich der Gesetzgebung und des Aufbaus der Rechtspflege. Ein neuer Schwerpunkt der Arbeit der IRZ-Stiftung ist u.a. Gewaltschutz.
Nach langen Diskussionen des Pro und Kontra auch schon in vorangegangenen Vorstandssitzungen fasst der Bundesvorstand am 19. Februar 2011 auch einen einstimmigen Beschluss, aus dem Deutschen Frauenrat zum Ende des Jahres 2011 auszutreten. Die Gründe sind im Wesentlichen im Bericht der Delegierten Ingeborg Heinze, djbZ 2/2011, S. 80 nachzulesen. Der Wiedereintritt für den Fall einer Satzungsbzw. Strukturänderung ist seitens des Bundesvorstands automatisch in vier Jahren (2015) zu prüfen. Von dieser Entscheidung nicht betroffen ist die vielfach erfreuliche Zusammenarbeit und Mitgliedschaft der Landesverbände des djb in den Landesfrauenräten, die vom Deutschen Frauenrat rechtlich und organisatorisch völlig unabhängige Vereine sind.

Am 25. Juni 2011 stand die Veranstaltungsplanung im Mittelpunkt. Es stehen viele Veranstaltungen an, darunter der Bundeskongress im September 2011, die diesjährige Veranstaltung zum Projekt „Aktionärinnen fordern Gleichberechtigung“ im November 2011, eine Veranstaltung anlässlich „10 Jahre djb in Berlin “ im März 2012, der Juristentag im September 2012, parallel dazu eine Veranstaltung „Juristinnen fragen, Politikerinnen und Politiker antworten “ in München, das djb-Seminar „10 Jahre Gewaltschutzgesetz“ im Oktober 2012 in Bonn und - auch hieran wird schon gearbeitet - der Bundeskongress im September 2013 in Leipzig. Aktuelle Informationen zu Programm - soweit es schon spruchreif ist und Anmeldung finden sich immer im Veranstaltungsbereich auf der djb-Webseite (http://www.djb.de/Veranstaltungen/). Über diese Planung hinaus haben wir die Einrichtung des Parlamentarischen Abends beschlossen, wie sie der DAV und der DRB schon lange anbieten. Meine Nachfolgerin im Amt wird sich mit der Umsetzung dieses Beschlusses befassen.

In beiden Sitzungen wurde von allen - wie immer - ausführlich über Teilnahme an Veranstaltungen, individuell geführte politische Gespräche, von den Kommissionsvorsitzenden über die laufende Arbeit, also aus der ganzen Fülle der Arbeit jedes Vorstandsmitglieds, einschließlich Presse- und Öffentlichkeitsarbeit berichtet.

Vieles davon, auch Arbeitsergebnisse in Form von djb-Stellungnahmen und -Pressemitteilungen können immer aktuell auf der Homepage des djb abgerufen werden. Wer laufend über neue Stellungnahmen, Pressemitteilungen und djb-Termine informiert werden möchte, kann diese als E-Mail abonnieren (http://www.djb.de/st-pm/).

In diesem Zusammenhang der Hinweis, dass unser eigenes djb-Forum mittlerweile online ist (http://www.djb.de/ mitgliederbereich/djb-Forum/) und ausschließlich den Mitgliedern des djb zur Verfügung steht. Es hat Funktionen wie andere Foren auch. Die Mitglieder können ihre Daten verwalten, bei Interesse mit anderen Mitgliedern in Kontakt treten, sich über Themen in Gruppen austauschen und vieles mehr. Probieren Sie es aus, für Kritik, Hinweise und Wünsche 
z.B. per E-Mail an die Geschäftsstelle (geschaeftsstelle@djb. de) sind wir dankbar.

„Geschlechtergerechte Sprache in djb-Publikationen “ war ein weiteres Thema am 25. Juni. Der Bundesvorstand hat seinen Beschluss vom 27. Oktober 2002, konsequent zu gendern, bestätigt. Diskutiert wurde über die Art und Weise. Erhellende Beiträge hat die Koordinationsstelle für Geschlechterstudien, Frauenforschung und Frauenförderung an der Karl-Franzens-Universität Graz veröffentlicht (http://www. uni-graz.at/kffwww/GeschlechtergerechteSprache.htm). Dies und ein Aufsatz „HERRliche deutsche Sprache?“ mit vielen Anregungen wird auf der Webseite zur Verfügung gestellt (http://www.uni-graz.at/uedo1www_files_geschlechtergerechtes_formulieren-4.pdf). Beschlusslage im djb ist nun: (1) wenn möglich ist auf geschlechtsbezogene Begriffe zu verzichten, (2) wenn das nicht geht, sind Frau und Mann zu nennen, und zwar in dieser Reihenfolge, (3) bei Abkürzungen sind Schrägstriche zu verwenden. Dies gilt für alle djb-Publikationen, so dass ich die Autorinnen bitte, darauf schon beim Schreiben der Beiträge zu achten.

Zum Schluss lade ich Sie ein, sich zu unserem 39. Bundeskongress vom 22. bis 25. September 2011 in Potsdam anzumelden (http://www.djb.de/Veranstaltungen/2011/2011
Kongress/). Das Thema „Gleichberechtigung im öffentlichen Dienst - Anspruch und Wirklichkeit" geht viele von uns an. Die „Gläserne Decke“ gibt es leider immer noch. Ziel der Tagung ist daher, die rechtlichen Rahmenbedingungen von weiblichen Beschäftigten im öffentlichen Dienst zu evaluieren, Empfehlungen für Verbesserungen des gleichstellungsrechtlichen Instrumentariums und Impulse für die Gleichstellungspolitik des Bundes und die Arbeit anderer Verbände und sonstiger in diesem Bereich engagierter Personen zu geben. Die Situation von Frauen in Unternehmen der privaten Rechtsform wird dabei einbezogen, insbesondere um zu überprüfen, ob sich einzelne Instrumente im öffentlichen Dienst auf privatwirtschaftliche Beschäftigungsverhältnisse übertragen lassen.

Junge Juristinnen sollten sich von den Kosten nicht abschrecken lassen. Es gibt die Möglichkeit, Patinnengeld zu beantragen. Bitte stellen Sie bis Ende August einen formlosen Antrag unter Angabe der zu erwartenden Kosten an die Geschäftsstelle (geschaeftsstelle@djb.de). Am Freitag, 23. September 2011 treffen sich die Jungen Juristinnen zum Austausch.

Wir sehen uns hoffentlich in Potsdam! Bis dahin bin ich zum letzten Mal an dieser Stelle - Ihre Jutta Wagner.

\section{Mitgliederversammlung am 24. September 2011, Potsdam Kandidaturen für den Bundesvorstand (Präsidium, Beisitzerinnen und Delegierte)}

Am 24. September 2011 werden in Potsdam das Präsidium, bis zu zwei Beisitzerinnen, die Delegierten des djb zur Wahrnehmung von Mitgliedschaftsrechten bei anderen Verbänden und zwei Kassenprüferinnen neu gewählt. Präsidentin Jutta Wagner und Vizepräsidentin Renate Maltry haben ihre maximale Amtszeit nach $\mathbb{\int} 7$ Absatz 2 der Satzung erreicht und können daher kein weiteres Mal kandidieren. Bei der Schatzmeisterin ist eine mehrfache Wiederwahl möglich, so dass Dagmar Brinkmann, die das Amt der Schatzmeisterin nun ebenfalls sechs Jahre ausübt, wieder kandidieren kann und auch signalisiert hat, dieses zu tun. Auch Katharina König, seit 2009 Beisitzerin als Vertreterin der Mitglieder in Ausbildung, steht nach Bestehen ihres ersten Juristischen Staatsexamens im Dezember 2010 für eine weitere Amtszeit zur Verfügung. Ihre bisherige Arbeit als Delegierte möchten gerne fortsetzen: Christa Seeliger (Deutsche Liga für das Kind und UN-Women) und Katharina Wolf (Netzwerk Europäische Bewegung Deutschland). Kandidaturen für alle Ämter sind noch bis zum Tag der Mitgliederversammlung möglich.

Ihre Kandidaturen haben angekündigt:

Für das Amt der Präsidentin:

Ramona Pisal, Jahrgang 1957, geboren und aufgewachsen im Rheinland. Studium der Rechtswissenschaften an den Univer- sitäten Gießen und Köln. Nach knapp einem Jahr als Rechtsanwältin Eintritt in den richterlichen Dienst des Landes Nordrhein-Westfalen. Einsatz als Richterin bei dem Landund Amtsgericht Mönchengladbach in Zivil- und Strafsachen. Mitte der 90er Jahre zunächst Abordnung an das Amtsgericht Potsdam, dort auch Pressesprecherin. Nach Abordnung an das Brandenburgische Oberlandesgericht 1997 Versetzung in den richterlichen Dienst des Landes Brandenburg und Ernennung zur Richterin am Oberlandesgericht, zusätzlich Pressesprecherin. Nach fünfjähriger Tätigkeit in einem Zivilsenat seit 2001 Angehörige des 2. Strafsenat, seit 2006 dessen Leitung als Vorsitzende Richterin am Oberlandesgericht. Seit 2002 Gleichstellungsbeauftragte des Brandenburgischen Oberlandesgerichts und seit Inkrafttreten dieses Gesetzes Beschwerdestelle nach dem AGG. Seit Dezember 2006 Vorsitzende des Landesverbandes Brandenburg im djb, seit September 2007 Vizepräsidentin im Bundesvorstand. Mitglied im Deutschen Juristentag (djt) und Deutschen Richterbund (DRB), verheiratet und Mutter eines erwachsenen Sohnes.

Frauen in Führungspositionen sind auch im öffentlichen Dienst und der Justiz nicht selbstverständlich, wie ich u.a. aus meiner Aufgabe als Gleichstellungsbeauftragte weiß. Den Gründen hierfür und Möglichkeiten zur Abhilfe gilt mein besonderes Interesse. 